Pacific Journal of Mathematics

ON THE BEST CONDITIONS ON THE GRADIENT OF
PRESSURE FOR UNIQUENESS OF VISCOUS FLOWS IN THE

GiovanNi P. GALDI AND SALVATORE RIoNero 


\title{
ON THE BEST CONDITIONS \\ ON THE GRADIENT OF PRESSURE \\ FOR UNIQUENESS OF VISCOUS FLOWS \\ IN THE WHOLE SPACE
}

\section{Giovanni P. Galdi and Salvatore Rionero}

\begin{abstract}
In this paper we prove a uniqueness theorem for the Cauchy problem of the Navier-Stokes equations under the assumption on the gradient of pressure $\nabla p$ that it either belongs to some $L^{q}$ space for some $q \in(1, \infty)$ or tends to zero at large spatial distances. As shown by means of a counterexample, in the class where uniqueness is proven the above hypotheses cannot be relaxed to $\nabla p$ only bounded.
\end{abstract}

Introduction. Let $\Omega$ be an unbounded domain of the Euclidean 3-dimensional space $E_{3}$. During the last years, the problem of uniqueness (and, more generally, of continuous dependence) for solutions to nonsteady Navier-Stokes equation in $\Omega$, whose (global) kinetic energy need not be a priori a finite quantity, has drawn the attention of many writers [1-10]. As is well known, this kind of question traces back to early papers of J. Kampé de Fériet [9], D. Graffi [6] and J. Serrin [12]. The aim of these works is to try to recover uniqueness without giving a priori on the solutions decay or summability conditions which for some applications must be considered restrictive from the physical point of view (cf. e.g. [9], pp. 21-24, [12], pp. 63-64)'.

The above results can be grouped, essentially, into two classes. Specifically, indicating by $\mathbf{v}$ and $p$ the velocity and pressure fields, respectively, we have

(i) Uniqueness theorems without assumptions on $p$ but with restrictive hypotheses (e.g. summability) on the behavior of $\mathbf{v}$ and, possibly, on its derivatives [1-3];

(ii) Uniqueness theorems with assumptions on $p$ (e.g. summability) but with very mild hypotheses (e.g. boundedness or even "growth" at large spatial distances) on $\mathbf{v}$ and, possibly, on its first spatial derivatives [3-10].

In this paper we investigate uniqueness in a (ii)-type class, namely, we consider the class $\mathcal{C}$ of solutions where $\nabla \mathbf{v}$ is bounded and try to determine the "best assumptions" on $\nabla p$ for uniqueness to hold. Precisely, we prove that, for the Cauchy problem in the class $\mathcal{C}$, uniqueness

\footnotetext{
${ }^{1}$ We should notice that here we are not interested in uniqueness of solutions which are assumed to be square summable together with their first spatial derivatives. For this type of problem the reader is referred, e.g., to [8] and the references cited therein.
} 
holds provided that either $\nabla p \in L^{q}\left(E_{3} \times[0, T]\right), 1<q<\infty$, or $\nabla p=$ $o(1)$ as $|x| \rightarrow \infty$. These assumptions on $\nabla p$ can be considered the best possible in the sense that uniqueness fails in the class $e$ if $\nabla p$ is only bounded, as can be proven by means of a counterexample.

The paper is subdivided into two sections. In $\S 1$, after briefly recalling some preliminaries, we state our thoerem and give a counterexample showing that in the class where $\mathbf{v}$ and $\nabla \mathbf{v}$ are bounded uniqueness fails if $\nabla p$ is only bounded too. In $\S 2$, employing the weight function approach [4-11] and some estimates concerning elliptic equations, we show that a motion $(\mathbf{v}, p)$ in $E_{3}$ with $\nabla \mathbf{v}$ bounded is unique in the class of motions $(\mathbf{v}+\mathbf{u}, p+\tau)$ such that $\nabla \mathbf{u}$ is bounded and either $\nabla \tau \in L^{q}\left(E_{3} \times[0, T]\right)$, $1<q<\infty$, or $\nabla \tau=o(1)$ as $|x| \rightarrow \infty$, which is just the theorem enunciated in $\S 1$.

1. Preliminaries and statement of the theorem. As is known, the motion of a viscous, homogeneous Newtonian fluid occurring in the domain $\Omega \subseteq E_{3}$, is governed by the following Navier-Stokes system (we assume, for the sake of simplicity, the kinetic viscosity to be equal to 1)

$$
\left\{\begin{array}{l}
\frac{\partial \mathbf{v}}{\partial t}+\mathbf{v} \cdot \nabla \mathbf{v}=-\nabla p+\triangle_{2} \mathbf{v}+\mathbf{f}, \\
\nabla \cdot \mathbf{v}=0, \\
\mathbf{v}(x, t)=\mathbf{v}^{*}(x, t), \quad(x, t) \in \partial \Omega \times[0, T], \\
\mathbf{v}(x, 0)=\mathbf{v}_{0}(x), \quad x \in \Omega,
\end{array}\right.
$$

where $\mathbf{v}$ is the velocity, $p$ is the pressure, $\mathbf{f}$ is the external force, and finally, $\mathbf{v}^{*}$ and $\mathbf{v}_{0}$ are ascribed functions. From (1) it comes out that the uniqueness of a given motion $(\mathbf{v}, p)$ in the class of motions $(\mathbf{v}+\mathbf{u}, p+\tau)$ is then reduced to investigate the uniqueness of the null solution of the following initial boundary value problem

$$
\begin{cases}\frac{\partial \mathbf{u}}{\partial t}+(\mathbf{v}+\mathbf{u}) \cdot \nabla \mathbf{u}=-\mathbf{u} \cdot \nabla \mathbf{v}-\nabla \boldsymbol{\tau}+\triangle_{2} \mathbf{u} \\ \nabla \cdot \mathbf{u}=0, & \\ \mathbf{u}(x, t)=0, & (x, t) \in \partial \Omega \times[0, T], \\ \mathbf{u}(x, 0)=0, & x \in \Omega .\end{cases}
$$

The aim of this paper is to prove the following theorem.

THEOREM. Let $\Omega \equiv E_{3}$ and $\nabla \mathbf{v}, \nabla \mathbf{u}$ uniformly bounded in $E_{3} \times[0, T]$. Then if either

$$
\nabla \tau \in L^{q}\left(E_{3} \times[0, T]\right) \quad(q \in(1, \infty))
$$


or

$$
\nabla \tau=o(1)
$$

the solution $\mathbf{u}(x, t)$ to problem (2) is identically zero in $E_{3} \times[0, T]$.

As we mentioned in the Introduction, the above assumptions on $\nabla \tau$ cannot be relaxed to $\nabla \tau$ only bounded. Actually, we have the following counterexample to uniqueness. Let us take in $(1)_{1} \mathbf{f}=0$ and $\Omega=E_{3}$. It can be readily seen that $\mathbf{v}=0, p=$ const and $\mathbf{v}^{\prime}=(\sin t, 0,0), p^{\prime}=$ $-x_{1} \cos t$ are two solutions to problem $(1)^{2}$ assuming the same initial data and, moreover, $\mathbf{v}, \mathbf{v}^{\prime}, \nabla \mathbf{v}, \nabla \mathbf{v}^{\prime}, \nabla\left(p-p^{\prime}\right)$ are only uniformly bounded in $E_{3} \times[0, T]$.

2. Proof of the uniqueness theorem. The proof of the theorem will be given in several steps. First of all we propose a quite general lemma concerning an $a$ priori estimate for regular solutions to problem (2) in unbounded domains. We have

LEMMA 1. Let $\Omega$ be any unbounded domain in $E_{3}$ whose (2-dimensional) boundary, if any, is sufficiently smooth to allow the application of the divergence theorem. Moreover, assume that with respect to a fixed spherical coordinates system $(r, \gamma)$ with the origin at a given point $O \in E_{3}$ it holds

$$
\begin{cases}v_{r}, u_{r}=O(r), \quad \mathbf{u}=O\left(r^{m}\right) & (m \geq 0), \\ \nabla \mathbf{v}=O(1), \quad \nabla \mathbf{u}=O\left(r^{k}\right) & (k \geq 0)\end{cases}
$$

where $v_{r}$ and $u_{r}$ are the radial components of $\mathbf{v}$ and $\mathbf{u}$ respectively. Then for $q \in(1, \infty)$

$$
\nabla \tau \in L^{q}(\Omega \times[0, T]) \Rightarrow \mathbf{u}(t) \in L^{q}(\Omega) \quad \forall t \in[0, T]
$$

and the following estimate holds $(u=|\mathbf{u}|)$

$$
\int_{\Omega} u^{q}(x, t) d x \leq A \int_{0}^{t} \int_{\Omega}|\nabla \tau|^{q} d x d s \quad \forall t \in[0, T]
$$

where $A$ is a positive constant independent of $t$.

\footnotetext{
${ }^{2}$ We would remark that these solutions are a particular case of the following general class of potential-like solutions to (1) in $E_{3}$ with $\mathbf{f}=0$ :

$$
\left\{\begin{array}{l}
\mathrm{v}(x, t)=a(t) \nabla \psi \\
p(x, t)=-\frac{d a}{d t} \psi+\left(a^{2} / 2\right)(\nabla \psi)^{2}+c(t)
\end{array}\right.
$$
}

where $a \in C^{1}(0, T), \triangle_{2} \psi=0$ and $c(t)$ is an arbitrary function of $t \in[0, T]$. 
Proof. We shall employ the so called "generalized weighted energy equality" which we already introduced in [11]. This inequality is obtained by multiplying both sides of $(2)_{1}$ by $g u^{q-2} \mathbf{u}(q>1)$, where $g$ is a rapidly decreasing (smooth) function (weight function) and integrating by parts over $\Omega$. Thus we have (cf. [11])

$$
\left\{\begin{array}{r}
\frac{d E^{(q)}}{d t}=\int_{\Omega}\left\{\frac{u^{(q)}}{q}\left[\frac{\partial g}{\partial t}+(\mathbf{u}+\mathbf{v}) \cdot \nabla g\right]\right. \\
-g u^{q-2}\left[\mathbf{u} \cdot \nabla \mathbf{v} \cdot \mathbf{u}+\nabla \mathbf{u}: \nabla \mathbf{u}+(q-2) u^{-2}(\nabla \mathbf{u} \cdot \mathbf{u})^{2}+\nabla \tau \cdot \mathbf{u}\right] \\
\left.+(1 / q) \triangle_{2} g u^{q}\right\} d x
\end{array}\right.
$$

with

$$
E^{(q)}=(1 / q) \int_{\Omega} g u^{q} d x
$$

Let us choose in (4) $g(x, t)=\exp \left[-\alpha\left(t+t_{0}\right)^{\beta} r\right]\left(\alpha, \beta, t_{0}>0\right)$. We easily have the following inequalities

$$
\begin{aligned}
& -g u^{q-2} \mathbf{u} \cdot \nabla \mathbf{v} \cdot \mathbf{u} \leq M g u^{q} \quad(M=\text { const }>0) ; \\
& -g u^{q-2} \nabla \tau \cdot \mathbf{u} \leq g(1 / q)|\nabla \tau|^{q}+g \frac{(q-1)}{q} u^{q} ;
\end{aligned}
$$

$$
(1 / q) \triangle_{2} g u^{q} \leq \alpha^{2}\left(T+t_{0}\right)^{2 \beta}(1 / q) g u^{q} ;
$$

(iv) $-u^{q-2}\left[\nabla \mathbf{u}: \nabla \mathbf{u}+(q-2) u^{-2}(\nabla \mathbf{u} \cdot \mathbf{u})^{2}\right] \leq 0, \quad q \in(1, \infty)$.

Moreover, by assumption there is a constant $M^{\prime}$ such that $\left|u_{r}+v_{r}\right| \leq$ $M^{\prime}(r+1)$. Thus,

$$
\left\{\begin{array}{r}
\frac{\partial g}{\partial t}+(\mathbf{u}+\mathbf{v}) \cdot \nabla g \leq g M^{\prime} \alpha\left(T+t_{0}\right)^{\beta} \\
+\alpha\left(t+t_{0}\right)^{\beta} g r\left[-\frac{\beta}{\left(t+t_{0}\right)}+M^{\prime}\right]
\end{array}\right.
$$

Choosing

$$
\beta \geq M^{\prime}\left(T+t_{0}\right)
$$

from (5) we then deduce

$$
\frac{\partial g}{\partial t}+(\mathbf{u}+\mathbf{v}) \cdot \nabla g \leq g M^{\prime} \alpha\left(T+t_{0}\right) .
$$


Therefore, collecting (i)-(v), from (4) we obtain

$$
\frac{d E^{(q)}}{d t} \leq c_{1}\left[E^{(q)}+\int_{\Omega}|\nabla \tau|^{q} d x\right]
$$

where $c_{1}$ is a suitable positive constant (with respect to $t$ ). Consequently, integrating (6) and letting $\alpha \rightarrow 0$ prove (3).

LEMMA 2. Let $\varphi$ be a classical solution to the problem (in $E_{3}$ )

$$
\triangle_{2} \varphi=\nabla \cdot \mathbf{f}
$$

with $\mathbf{f}$ belonging to $L^{q}\left(E_{3}\right)$, for some $q \in(1, \infty)$. Assume that either

$$
\nabla \varphi \in L^{q}\left(E_{3}\right)
$$

or

$$
\nabla \varphi=o(1) \quad \text { as }|x| \rightarrow \infty,
$$

then it follows that in both cases (8), (9) $\nabla \varphi \in L^{q}\left(E_{3}\right)$ and the following estimate holds

$$
\int_{E_{3}}|\nabla \varphi|^{q} d x \leq C \int_{E_{3}}|\mathbf{f}|^{q} d x \quad(C=\text { const }>0) .
$$

Proof. We notice that, given $\mathbf{f} \in L^{q}\left(E_{3}\right)$, it is possible to prove the existence of a weak solution to (7) satisfying (10), i.e., of a function $\bar{\varphi}$ with $\nabla \bar{\varphi} \in L^{q}\left(E_{3}\right)$ such that

$$
\left\{\begin{array}{l}
\int_{E_{3}} \nabla \bar{\varphi} \cdot \nabla \psi d x=\int_{E_{3}} \mathbf{f} \cdot \nabla \psi d x \\
\int_{E_{3}}|\nabla \bar{\varphi}|^{q} d x \leq C \int_{E_{3}}|\mathbf{f}|^{q} d x,
\end{array}\right.
$$

where $\psi$ is an arbitrary (measurable) function with $\nabla \psi \in L^{q^{\prime}}\left(E_{3}\right)\left(q^{\prime}=\right.$ $q /(q-1))$ and $C$ is a positive constant ${ }^{3}$. On the other hand, since $\varphi$ is a classical solution to (7), from (7) and (11), we must have

$$
\int_{E_{3}} \nabla w \cdot \nabla \psi^{*} d x=0
$$

\footnotetext{
${ }^{3}$ In fact, if $\mathbf{f} \in C_{0}^{\infty}\left(E_{3}\right)$ we have $\bar{\varphi}(x)=(1 / 4 \pi) \int_{E_{3}} \nabla_{x}(1 /|x-y|) \cdot \mathbf{f} d y$. If $\mathbf{f} \in L^{q}\left(E_{3}\right)$ we can approximate it with smooth functions of compact support and then apply the well-known Calderon-Zygmund theorem on the boundedness in $L^{q}$ of the singular integrals.
} 
where $w=\varphi-\bar{\varphi}$ and $\psi^{*}$ is arbitrary from $C_{0}^{\infty}\left(E_{3}\right)$, so that $w$ is a weak solution to $\triangle_{2} w=0$ in $E_{3}$. From well-known results on the regularity of weak solutions to elliptic equations we then deduce that $w$ is in fact a classical solution to $\triangle_{2} w=0$ in $E_{3}$. Now, fix any point $x_{0} \in E_{3}$ and choose $x_{0}$ as the origin of a spherical coordinates system $(R, \theta)$. By using the Hölder inequality, it can be readily seen that from either (8) or (9) and from $(11)_{2}$ it turns out the existence of a sequence of radii $\left\{R_{n}\right\}_{n \in N}$ starting from $x_{0}$ with $\lim _{n \rightarrow \infty} R_{n}=\infty$ such that

$$
\lim _{n \rightarrow \infty} \int_{S_{1}} \nabla w\left(R_{n}, \theta\right) d \theta=0,
$$

where $S_{1}$ is the unit sphere centered at $x_{0}$. Therefore, by the mean value theorem for harmonic functions applied to $\nabla w\left(x_{0}\right)$ we deduce $\nabla w\left(x_{0}\right)=$ $0 \forall x_{0} \in E_{3}$. This fact, in virtue of $(11)_{2}$, completely proves the lemma.

We are now in a position to prove the theorem. From $\nabla \cdot \mathbf{u}=\nabla \cdot \mathbf{v}$ $=0$, one obtains the obvious identity

$$
\nabla \cdot(\mathbf{v} \cdot \nabla \mathbf{u})=\nabla \cdot(\mathbf{u} \cdot \nabla \mathbf{v}) .
$$

As a consequence, taking the divergence operator of both sides of (2) we have that at each $t \in[0, T] \tau$ satisfies

$$
\left\{\begin{array}{l}
\triangle_{2} \tau=\nabla \cdot \boldsymbol{\Psi} \\
\boldsymbol{\Psi}=-\mathbf{u} \cdot(\nabla \mathbf{u}+2 \nabla \mathbf{v}) .
\end{array}\right.
$$

From the assumptions of the theorem and from Lemma 2 we then deduce the existence of a constant $B$ such that

$$
\int_{0}^{t} \int_{E_{3}}|\nabla \tau|^{q} d x d s \leq B \int_{0}^{t} \int_{E_{3}} u^{q} d x d s \quad \forall t \in[0, T] .
$$

On the other hand, given $w \in C^{1}\left(E_{3} \times[0, T]\right)$ with $\sup _{E_{3} \times[0, T]}|\nabla w|=$ $N<\infty$, it is

$$
|w(x, t)| \leq N r+|w(0, T)| \leq N r+N^{\prime}
$$

where $N^{\prime}=\max _{t \in[0, T]}|w(0, t)|$. Consequently, by the assumptions of the theorem we have

$$
\sup _{E_{3} \times[0, T]}|\mathbf{u} /(1+r)|, \quad \sup _{E_{3} \times[0, T]}|\mathbf{v} /(1+r)|<\infty .
$$

Thus, employing (14), Lemma 1 and (13) we finally obtain $\forall t \in[0, T]$

$$
\int_{E_{3}} u^{q}(x, t) d x \leq D \int_{0}^{t} \int_{E_{3}} u^{q}(x, t) d x d s \quad(D=\text { const }>0)
$$

which in turn implies $\mathbf{u}(x, t) \equiv 0$ in $E_{3} \times[0, T]$. 


\section{REFERENCES}

1. D. Edmunds, On the uniqueness of viscous flows, Arch. Rational Mech. Anal., 14 (1963), 171-176.

2. E. B. Fabes, B. F. Jones \& N. M. Riviere, The initial value problem for the Navier-Stokes equations with data in $L^{p}$, Arch. Rational Mech. Anal., 45 (1972), 222-240.

3. M. Fabrizio, Problemi di unicita' per le equazioni di Navier-Stokes in domini non limitati, Arch. Rational Mech. Anal., 68 (1979), 171-178.

4. G. P. Galdi \& S. Rionero, A uniqueness theorem for hydrodynamic flows in unbounded domains, Ann. Mat. Pura App., 108 (1976), 361-366.

5. Continuous dependence theorems for Navier-Stokes equations in unbounded domains by the weight function method, Q. J1. Mech. Appl. Math., 32 (1979), 149-161.

6. D. Graffi, Sul teorema di unicita' nella dinamica del fluidi, Ann. Mat. Pura App., 50 (1960), 379-388.

7. J. G. Heywood, A uniqueness theorem for non-stationary Navier-Stokes equations past an obstacle, Annali Sc. Norm. Pisa, Ser. IV, 6 (3) (1979), 427-444.

8. , On uniqueness questions in the theory of viscous flow, Acta Mathematica, 136 (1976), 61-102.

9. J. Kampé de Fériet, Problèmes Mathématiques de la théorie de la turbulence homogène, Corso C.I.M.E. sulla "Teoria della Turbolenza", Varenna (Italy), Libreria Universitaria Levrotto \& Bella-Torino, (1957), 1-104.

10. S. Rionero \& G. P. Galdi, On the uniqueness of viscous fluid motions, Arch. Rational Mech. Anal., 62 (1976), 295-301.

11. The weight function approach to uniqueness of viscous flows in unbounded domains, Arch. Rational Mech. Anal., 69 (1979), 37-52.

12. J. Serrin, Comparison and averaging method in mathematical physics, Corso C.I.M.E. su "Proprieta' di media e teoremi di confronto in fisica matematica", Bressanone (Italy), Edizioni Cremonese-Roma, (1963), 1-87.

13. B. Straughan, Uniqueness and continuous dependence theorems for the conduction diffusion solution to the Boussinesq equations on an exterior domain, J. Math. Anal. App., 57 (1977), 203-234.

Received July 9, 1980. The research described in this paper was made under the auspices of G.N.F.M. of the Italian C.N.R.

UNIVERSITA' DI NAPOLI

"RENATO CACCIOPPOLl"

Via MEZzocannone, 8

CAP. 80134, NAPOLI, ITALY 



\section{PACIFIC JOURNAL OF MATHEMATICS \\ EDITORS}

DONALD BABBITT (Managing Editor)

University of California

Los Angeles, CA 90024

\section{Hugo Rossi}

University of Utah

Salt Lake City, UT 84112

C. C. Moore and Arthur Ogus

University of California

Berkeley, CA 94720
J. DugundiI

Department of Mathematics

University of Southern California

Los Angeles, CA 90089-1113

R. FINN and H. SAMELSON

Stanford University

Stanford, CA 94305

ASSOCIATE EDITORS
R. ARens
E. F. BECKENBACH
B. H. NeumanN
F. WOLF
K. YoshidA (1906-1982)

\section{SUPPORTING INSTITUTIONS}

UNIVERSITY OF ARIZONA

UNIVERSITY OF BRITISH COLUMBIA

CALIFORNIA INSTITUTE OF TECHNOLOGY

UNIVERSITY OF CALIFORNIA

MONTANA STATE UNIVERSITY

UNIVERSITY OF NEVADA, RENO

NEW MEXICO STATE UNIVERSITY

OREGON STATE UNIVERSITY
UNIVERSITY OF OREGON

UNIVERSITY OF SOUTHERN CALIFORNIA

STANFORD UNIVERSITY

UNIVERSITY OF HAWAII

UNIVERSITY OF TOKYO

UNIVERSITY OF UTAH

WASHINGTON STATE UNIVERSITY

UNIVERSITY OF WASHINGTON 


\section{Pacific Journal of Mathematics}

Vol. 104, No. 1

May, 1983

Nestor Edgardo Aguilera and Eleonor Ofelia Harboure de Aguilera, On

the search for weighted norm inequalities for the Fourier transform $\ldots \ldots .1$

Jin Akiyama, Frank Harary and Phillip Arthur Ostrand, A graph and its complement with specified properties. VI. Chromatic and achromatic numbers ......................................... 15

Bing Ren Li, The perturbation theory for linear operators of discrete type . . . 29

Peter Botta, Stephen J. Pierce and William E. Watkins, Linear

transformations that preserve the nilpotent matrices .............. 39

Frederick Ronald Cohen, Ralph Cohen, Nicholas J. Kuhn and Joseph

Alvin Neisendorfer, Bundles over configuration spaces .......... 47

Luther Bush Fuller, Trees and proto-metrizable spaces . . . . . . . . . . 55

Giovanni P. Galdi and Salvatore Rionero, On the best conditions on the

gradient of pressure for uniqueness of viscous flows in the whole space . . 77

John R. Graef, Limit circle type results for sublinear equations $\ldots \ldots \ldots \ldots 85$

Andrzej Granas, Ronald Bernard Guenther and John Walter Lee,

Topological transversality. II. Applications to the Neumann problem for

$y^{\prime \prime}=f\left(t, y, y^{\prime}\right) \ldots \ldots \ldots \ldots \ldots \ldots \ldots \ldots \ldots \ldots \ldots \ldots \ldots \ldots \ldots . \ldots 5$

Richard Howard Hudson and Kenneth S. Williams, Extensions of

theorems of Cunningham-Aigner and Hasse-Evans . . . . . . . . . . . 111

John Francis Kurtzke, Jr., Centralizers of irregular elements in reductive algebraic groups

James F. Lawrence, Lopsided sets and orthant-intersection by convex

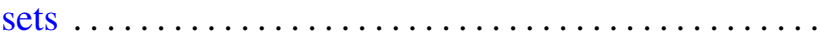

Åsvald Lima, G. H. Olsen and U. Uttersrud, Intersections of $M$-ideals and

$G$-spaces

Wallace Smith Martindale, III and C. Robert Miers, On the iterates of derivations of prime rings

Thomas H. Pate, Jr, A characterization of a Neuberger type iteration procedure that leads to solutions of classical boundary value problems

Carl L. Prather and Ken Shaw, Zeros of successive iterates of multiplier-sequence operators

Billy E. Rhoades, The fine spectra for weighted mean operators

Rudolf J. Taschner, A general version of van der Corput's difference theorem

Johannes A. Van Casteren, Operators similar to unitary or selfadjoint ones 